Exciton transport into a single GaAs quantum wire studied by picosecond near-field optical spectroscopy

A. Richter, M. Süptitz, D. Heinrich, Ch. Lienau, T. Elsaesser, M. Ramsteiner, R. Nötzel, and K. H. Ploog

Citation: Appl. Phys. Lett. 73, 2176 (1998); doi: 10.1063/1.122414

View online: http://dx.doi.org/10.1063/1.122414

View Table of Contents: http://aip.scitation.org/toc/apl/73/15

Published by the American Institute of Physics

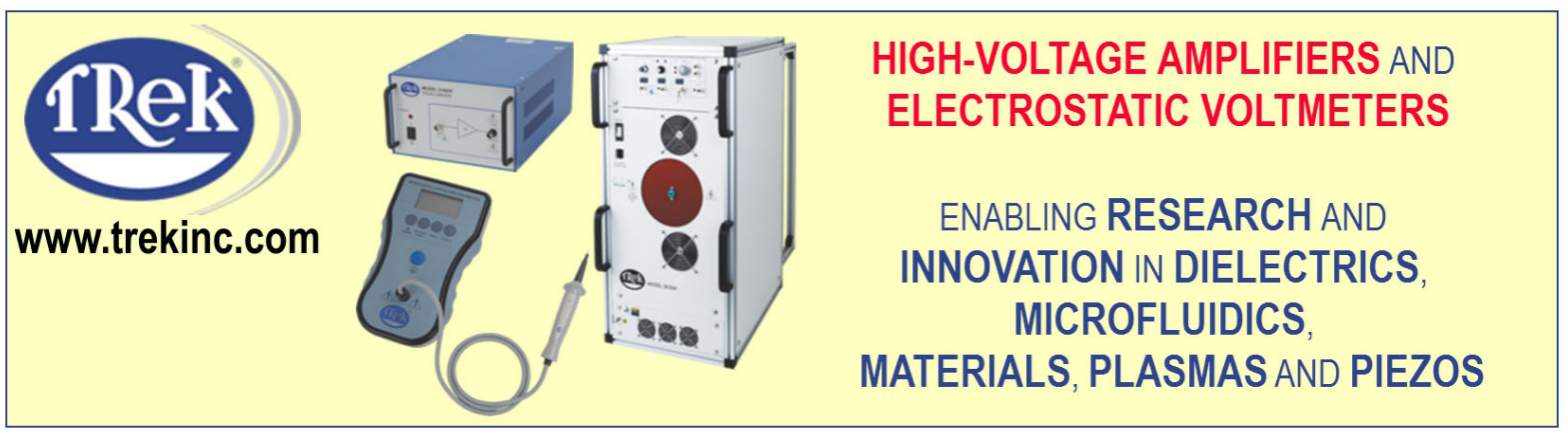




\title{
Exciton transport into a single GaAs quantum wire studied by picosecond near-field optical spectroscopy
}

\author{
A. Richter, M. Süptitz, D. Heinrich, Ch. Lienau, ${ }^{\text {a) }}$ and T. Elsaesser \\ Max-Born-Institut für Nichtlineare Optik und Kurzzeitspektroskopie, D-12489 Berlin, Germany \\ M. Ramsteiner, R. Nötzel, and K. H. Ploog \\ Paul-Drude-Institut für Festkörperelektronik, D-10117 Berlin, Germany
}

(Received 6 April 1998; accepted for publication 11 August 1998)

\begin{abstract}
We report a time-resolved near-field luminescence study of excitonic real-space transfer into single GaAs quantum wires. Excitons generated by local optical excitation in a $250 \mathrm{~nm}$ spot undergo diffusive transport over a length of several microns and are subsequently trapped into the quantum wire by optical phonon emission. Local energy barriers in the vicinity of the quantum wire, originating from the epitaxial growth mechanism of the nanostructure, directly influence the real-space transfer dynamics and trapping efficiency. (c) 1998 American Institute of Physics.
\end{abstract} [S0003-6951(98)04941-9]

Real-space transfer and trapping of carriers in semiconductor nanostructures are of particular interest both from the viewpoint of fundamental physics as well as for device applications. Submicron dimensions of devices and ultrashort scattering times of carriers require very high spatial and temporal resolution to study the microscopic mechanisms of such processes. Near-field scanning optical microscopy $(\mathrm{NSOM})^{1,2}$ allows one to overcome the diffraction-limited resolution of classical microscopy and offers subwavelength spatial resolution in the $100 \mathrm{~nm}$ range. In combination with time-resolved excitation and detection schemes it gives direct access to carrier dynamics on ultrashort time and length scales. This has been demonstrated in spatially resolved pump-probe experiments on GaAs microdisks ${ }^{3}$ and in a study of exciton spin dynamics in magnetic heterostructures. ${ }^{4}$

In this letter, we combine low-temperature near-field scanning optical microscopy and time-resolved luminescence spectroscopy to study real-space transfer of excitons and the subsequent trapping into a single quasi-one-dimensional nanostructure, a quantum wire (QWR). The high spatial resolution of $250 \mathrm{~nm}$ and the time resolution of $260 \mathrm{ps}$ allow a detailed analysis of the microscopic carrier dynamics in real space. We demonstrate that local energy barriers originating from QWR formation in the epitaxial growth process have strong influence on the transport properties of QWR structures.

We studied the QWR structure depicted schematically in Fig. 1(a). The sample was grown by molecular beam epitaxy on patterned GaAs (311)A substrates at the sidewall of 1520-nm-high mesa stripes oriented along [01-1]. ${ }^{5}$ It consists of a nominally 6-nm-thick GaAs quantum well (QW) layer clad by 50 -nm-thick $\mathrm{Al}_{0.5} \mathrm{Ga}_{0.5}$ As barriers. In the growth process, formation of a sidewall QWR arises from the preferential migration of $\mathrm{Ga}$ atoms within the QW layer from both the mesa top and the mesa bottom towards the sidewall, resulting in a local increase of the GaAs QW thickness. This

${ }^{\text {a)} C o r r e s p o n d i n g ~ a u t h o r: ~ E l e c t r o n i c ~ m a i l: ~ l i e n a u @ ~ m b i-b e r l i n . d e ~}$ change in QW thickness leads to a quasi-one-dimensional (1D) confinement over a lateral width of $50 \mathrm{~nm}$. The lateral confinement potential of this QWR plotted in Fig. 1(a) was derived from low-temperature photoluminescence excitation measurements with a spatial resolution of $250 \mathrm{~nm} .{ }^{6,7}$ These
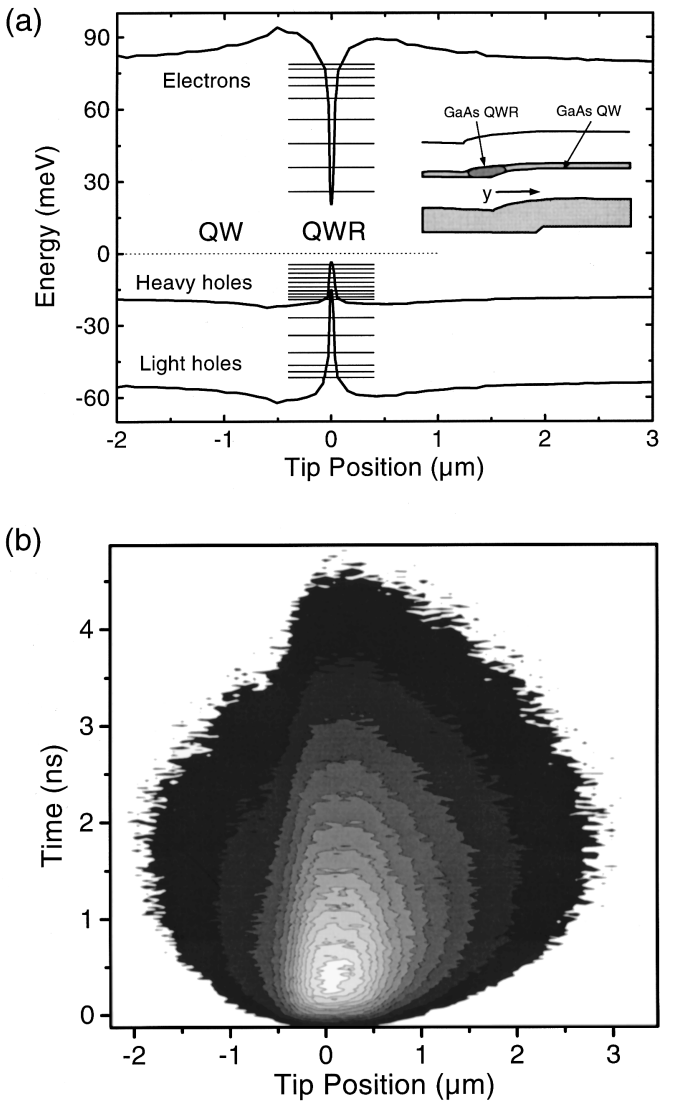

FIG. 1. (a) Lateral confinement potential of the QWR structure, as derived from spatially resolved photoluminescence excitation measurements. The horizontal lines indicate the calculated subband energies. Inset: schematic of the QWR structure. (b) Spatially and temporally resolved photoluminescence from the QWR after localized excitation in the embedding quantum well $\left(E_{\mathrm{ex}}=1.614 \mathrm{eV}\right.$, detection energy $\left.1.54 \mathrm{eV}\right)$. The luminescence intensity is plotted as a function of the lateral distance $y$ between excitation and QWR position and as a function of time. 
experiments performed with the same near-field probes as used in the present study, give local variations of the band gap of the embedding QW along the $y$ direction, i.e., perpendicular to the QWR. On the mesa bottom $(y<0)$, the QW band gap increases by up to $18 \mathrm{meV}$ over a length of $2 \mu \mathrm{m}$, representing a barrier for lateral carrier motion. On the mesa top $(y>0)$ the band gap shift is smaller and amounts to about $14 \mathrm{meV}$. The maxima of the two barriers separating QWR and flat area QW have a lateral distance of $800 \mathrm{~nm}$. The change in band gap energy originates from a local change in QW thickness. ${ }^{7}$ Within the experimental accuracy, the same spatial band gap profile was found for different positions on the sample.

In this structure, carrier transport is studied by timeresolved near-field spectroscopy using a low-temperature NSOM. ${ }^{8}$ Carriers locally excited in the QW by a short optical pulse undergo real-space transfer to the QWR and are trapped into 1D states. The dynamics of transport and trapping are monitored via the time-resolved onset of QWR luminescence. Spatial resolution is provided by transmitting femtosecond excitation pulses from a mode-locked Ti:sapphire laser through a metal-coated fiber probe of $200 \mathrm{~nm}$ diameter. ${ }^{9}$ The $16 \mathrm{meV}$ broad spectrum of the laser is centered at $1.614 \mathrm{eV}$ and overlaps the excitonic absorption band of the QW. QWR photoluminescence (PL) at $1.54 \mathrm{eV}$, the maximum of the QWR PL spectrum, is collected in the far field by a microscope objective. The PL is dispersed in a $0.25 \mathrm{~m}$ double monochromator (resolution $1.2 \mathrm{~nm}$ ) and detected with a silicon avalanche photodiode using timecorrelated single photon detection with a temporal resolution of 260 ps. Luminescence decay curves are accumulated for each of 100 excitation positions while scanning the fiber probe perpendicular to the wire axis. The laser power on the sample was 10 to $100 \mathrm{nW}$, corresponding to very low excitation densities between $10^{4}$ and $10^{5} \mathrm{~cm}^{-1}$.

In Fig. 1(b), the intensity of the time-resolved QWR emission at a sample temperature of $100 \mathrm{~K}$ is shown as a function of tip position along the lateral $y$ axis (abscissa) and delay time (ordinate). The QWR is located at $y=0$. Cross sections through Fig. 1(b), i.e., the time evolution of luminescence for fixed excitation position $|y|=0,0.4,1$, and 2 $\mu \mathrm{m}$ are shown in Figs. 2(a) and 2(b) for excitation on mesa top and mesa bottom, respectively. For excitation at $y=0$, we find a rise of the QWR luminescence that is limited by the temporal resolution of our experiment of $260 \mathrm{ps}$ and a decay of the QWR luminescence that is single exponential over two orders of magnitude with a decay time of $1.5 \mathrm{~ns}$. This temporal dependence of the QWR luminescence remains unchanged for $|y|<320 \mathrm{~nm}$, i.e., for excitation between the barriers separating QWR and embedding QW. For a distance $|y|>320 \mathrm{~nm}$, the onset of QWR luminescence is delayed with respect to that for excitation at $y=0$. The rise of the QWR luminescence becomes monotoneously slower with increasing $|y|$, as is evident from the shift of the maximum PL intensity in time. In Fig. 3, we plot the temporal position of the maximum PL intensity, $t_{\max }-t_{0}$, as a function of the excitation position $y\left(t_{0} \approx 0.5 \mathrm{~ns}\right.$ : maximum position for $|y|<0.32 \mu \mathrm{m})$. The value of $t_{\max }-t_{0}$ increases almost linearly with increasing distance $y$, with a slope of 0.73 $\mathrm{ns} / \mu \mathrm{m}$ for $y<0$ and $0.62 \mathrm{~ns} / \mu \mathrm{m}$ for $y>0$. The maximum
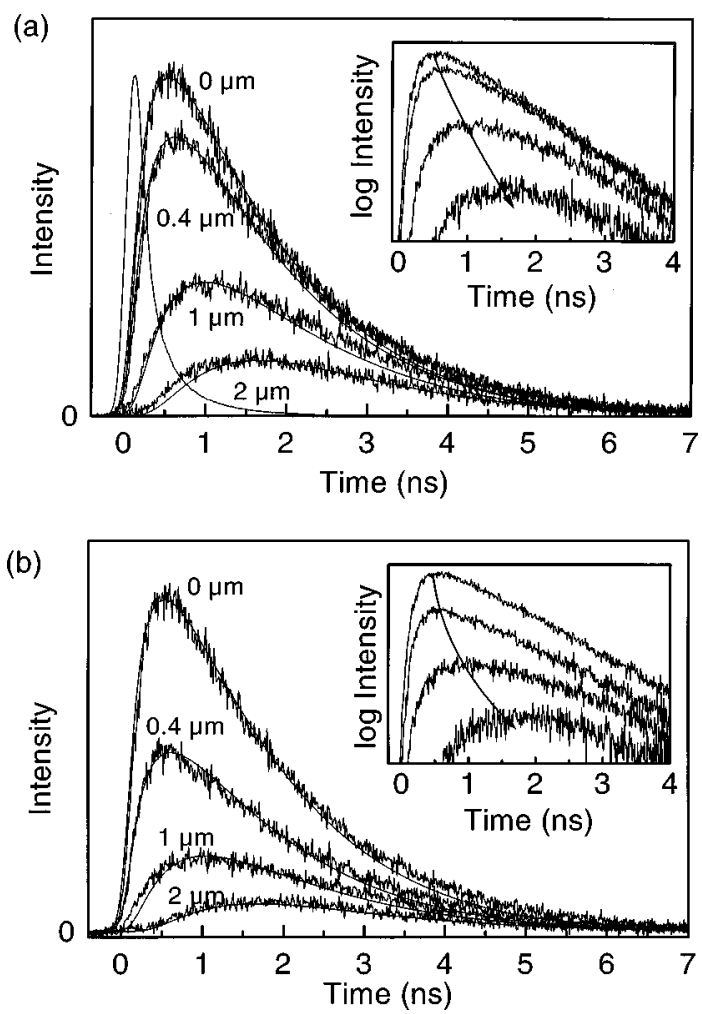

FIG. 2. Time evolution of QWR luminescence after excitation on the (a) mesa top and (b) mesa bottom of the QWR structure. The luminescence intensity at $1.54 \mathrm{eV}$ is plotted versus time for different excitation positions $y$ and shows an increasingly delayed rise with increasing $y$ which is due to the transport time of excitons from the excitation spot to the QWR. Insets: Data plotted on a logarithmic intensity scale. Solid lines: results of a numerical calculation based on a drift-diffusion model for exciton transport.

intensity of QWR luminescence after excitation on the mesa bottom $(y<0)$ is smaller than for mesa top $(y>0)$ excitation, i.e., fewer carriers are trapped into the QWR. This is evident from the amplitude values in Figs. 2(a) and 2(b) and from the shape of the plot in Fig. 1(b) at positive and negative $y$.

In the following, we discuss the luminescence behavior found for different excitation positions $y$. For $|y|$

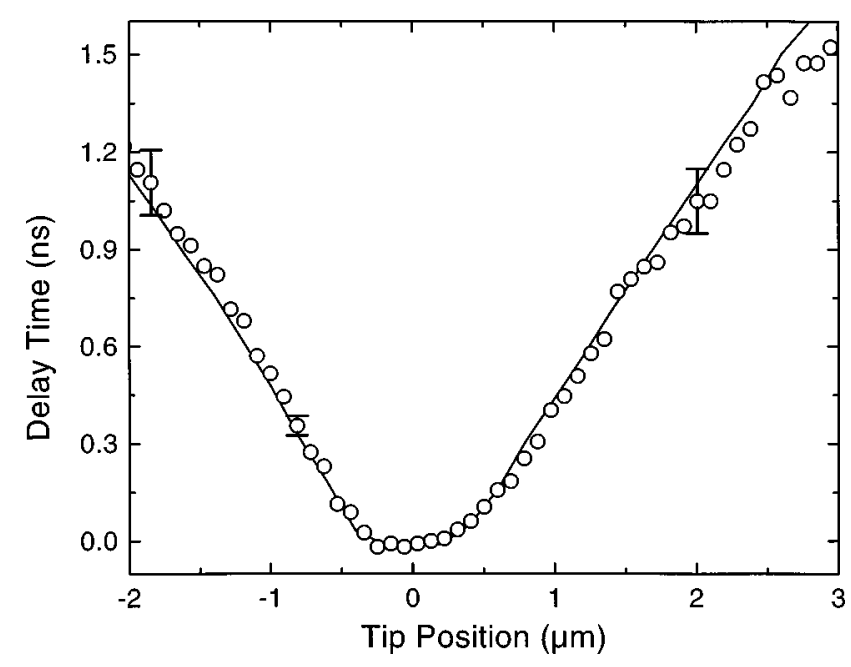

FIG. 3. Temporal position of the luminescence maxima of Fig. 2 as a function of the lateral separation $y$ between excitation spot and QWR (symbols). Solid line: Temporal positions calculated from the drift-diffusion model for exciton transport. 
$<0.32 \mu \mathrm{m}$, the single exponential decay of the QWR PL gives a population lifetime of the $\mathrm{QWR}^{10}$ of $1.5 \mathrm{~ns}$, slightly longer than the PL decay time of the embedding QW of 1.35 ns. The slow single exponential decay of the QWR PL indicates the high structural quality of the QWR sample. The fast rise of the QWR PL after excitation at $|y|<0.32 \mu \mathrm{m}$ demonstrates that carrier relaxation from continuum states into localized QWR states occurs within the time resolution of the experiment of $260 \mathrm{ps}$. This relaxation involves both trapping into and energy relaxation within the QWR. For our very low excitation density of $\leqslant 10^{5} \mathrm{~cm}^{-1}$, carrier-carrier scattering is negligible and emission of longitudinal optical phonons represents the dominant process populating lowlying QWR states. ${ }^{11}$

The onset of the QWR luminescence for excitation at $|y|>0.32 \mu \mathrm{m}$, i.e., for photogeneration of carriers in the QW, occurs with a delay of several hundred picoseconds. This delay increases with $y$ (Fig. 3), reflecting the traveling time of carriers from the excited QW area to the QWR location. For a lattice temperature of $100 \mathrm{~K}$ and weak excitation, real-space transfer in the $\mathrm{QW}$ is dominated by excitonic transport. ${ }^{12}$ The time scale of transport is several hundred picoseconds, much longer than the formation and energy relaxation times of excitons, i.e., excitons undergoing realspace transfer form a quasiequilibrium energy distribution close to lattice temperature. In such a case, an isothermal drift-diffusion model is appropriate to describe the spatially resolved exciton dynamics.

Exciton transport is described by the two-dimensional particle current density $\mathbf{j}(\mathbf{r}, t)=\mathbf{j}_{\text {diff }}(\mathbf{r}, t)+\mathbf{j}_{\text {drift }}(\mathbf{r}, t)$ with a diffusion term $\mathbf{j}_{\text {diff }}(\mathbf{r}, t)=-D_{\text {ex }} \nabla n(\mathbf{r}, t)$ induced by the gradient of the exciton concentration $n(\mathbf{r}, t)\left[\mathbf{r}=(x, y), D_{\mathrm{ex}}\right.$ : exciton diffusion coefficient] and a drift term $\mathbf{j}_{\text {drift }}(\mathbf{r}, t)$ $=-\mu_{\mathrm{ex}} n(\mathbf{r}, t) \nabla U(\mathbf{r})$ induced by the action of the local band gap gradient $\boldsymbol{\nabla} U(\mathbf{r})$ on the center of mass motion of the exciton $\left(\mu_{\mathrm{ex}}\right.$ : equivalent charged particle mobility of the exciton). ${ }^{13}$ Under the conditions of our experiment, $\mu_{\mathrm{ex}}$ is linked to the to the diffusion coefficient $D_{\mathrm{ex}}$ by the Einstein relation $\mu_{\mathrm{ex}}=e D_{\mathrm{ex}} / k T$ ( $e$ : electron charge, $k$ Boltzmann constant). The spatiotemporal evolution of the exciton concentration $n(\mathbf{r}, t)$ is described by the two dimensional continuity equation, including a generation, a diffusion, a drift, and a recombination term:

$$
\begin{aligned}
\frac{\partial n(\mathbf{r}, t)}{\partial t}= & g\left(\mathbf{r}, \mathbf{r}_{0}, t\right)+D_{\mathrm{ex}} \Delta n(\mathbf{r}, t)+\mu_{\mathrm{ex}} n(\mathbf{r}, t) \Delta U(\mathbf{r}) \\
& +\mu_{\mathrm{ex}} \boldsymbol{\nabla} n(\mathbf{r}, t) \boldsymbol{\nabla} U(\mathbf{r})-n(\mathbf{r}, t) / \tau(\mathbf{r}) .
\end{aligned}
$$

Here, the spatial variation of the band gap $U(\mathbf{r})$ along the lateral $y$ direction, perpendicular to the wire axis, is directly taken from the confinement potential in Fig. 1. Note that $U(\mathbf{r})$ does not depend on the $x$ coordinate, i.e., the direction parallel to the wire axis. The exciton lifetime $\tau(\mathbf{r})$ has a value of $1.5 \mathrm{~ns}$ for the QWR and $1.35 \mathrm{~ns}$ for the QW. For the generation term $g(\mathbf{r}, t)$, we use a Gaussian shape in time [full width at half maximum (FWHM) of $1 \mathrm{ps}]$ and space (FWHM of $300 \mathrm{~nm})$ centered at $\mathbf{r}=(0, y)$. The intensity of the timeresolved QWR luminescence is proportional to $I(t)$ $=\int d x \int d y[\partial n(\mathbf{r}, t) / \partial t]$, where the integration is performed over the length of the QWR along the $x$ axis and over the width of the QWR region along the $y$ axis. For comparison with experiment, $I(t)$ is convoluted with the temporal response function of the photodetector [solid line in Fig. 2(a)]. Assuming an exciton diffusion coefficient $D_{\mathrm{ex}}=13 \mathrm{~cm}^{2} / \mathrm{s}$, the calculated temporal dependence of the QWR luminescence is in good agreement for excitation on both mesa top and bottom (Fig. 2). This diffusion coefficient corresponds to an excitonic mobility $\mu_{\mathrm{ex}}=1500 \mathrm{~cm}^{2} / \mathrm{V} \mathrm{s}$ in the $\mathrm{QW}$, given mainly by the $2 \mathrm{D}$ hole mobility and limited in this temperature range by LO phonon scattering. In particular, the model calculation correctly describes the influence of the lateral band gap variation $U(\mathbf{r})$ on the exciton transport. In the region outside the barriers, $U(\mathbf{r})$ exerts a force on the excitonic center of mass motion that opposes the diffusive real space transfer towards the QWR. This is manifested in the experimental data as (i) a significantly weaker QWR luminescence for mesa bottom $(y<0)$ than mesa top $(y>0)$ excitation due to the higher barrier on the mesa bottom, and (ii) a slight shift of the temporal position of the maximum of the QWR emission $t_{\max }$ to shorter delay times on the mesa top. Both on the mesa top and bottom, the calculated $t_{\max }$ are in good agreement with the measured values (see Fig. 3), indicating that the exciton diffusion constants are similar on both sides of the QWR. We note that the influence of the drift current relative to the diffusion current becomes even more important at lower temperatures $(T=10 \mathrm{~K})$ where exciton transfer from outside the barriers into the QWR is fully suppressed. ${ }^{7}$

In conclusion, we demonstrated the pronounced influence of local, subwavelength variations of the band gap energy on the real-space exciton transport into single GaAs quantum wires. Such growth-correlated variations of the band gap potential on a submicron length scale determine the carrier dynamics in a wide variety of low-dimensional semiconductor nanostructures and time-resolved near-field spectroscopy presents a powerful tool for mapping these dynamics in both time and space.

Financial support by the Deutsche Forschungsgemeinschaft (SFB 296) is gratefully acknowledged.

${ }^{1}$ D. W. Pohl, W. Denk, and M. Lanz, Appl. Phys. Lett. 44, 651 (1984).

${ }^{2}$ E. Betzig and J. K. Trautman, Science 257, 189 (1992).

${ }^{3}$ J. B. Stark, U. Mohideen, E. Betzig, and R. E. Slusher, in Ultrafast Phenomena IX (Springer, Berlin, 1996), p. 349.

${ }^{4}$ J. Levy, V. Nikitin, J. M. Kikkawa, A. Cohen, N. Samarth, R. Garcia, and D. D. Awschalom, Phys. Rev. Lett. 76, 1948 (1996).

${ }^{5}$ R. Nötzel, M. Ramsteiner, J. Menniger, A. Trampert, H.-P. Schönherr, L. Däweritz, and K. H. Ploog, Jpn. J. Appl. Phys., Part 2 35, L297 (1996).

${ }^{6}$ A. Richter, G. Behme, M. Süptitz, Ch. Lienau, T. Elsaesser, M. Ramsteiner, R. Nötzel, and K. H. Ploog, Phys. Rev. Lett. 79, 2145 (1997).

${ }^{7}$ Ch. Lienau, A. Richter, G. Behme, M. Süptitz, D. Heinrich, T. Elsaesser, M. Ramsteiner, R. Nötzel, and K. H. Ploog, Phys. Rev. B 58, 2045 (1998).

${ }^{8}$ G. Behme, A. Richter, M. Süptitz, and C. Lienau, Rev. Sci. Instrum. 68, 3458 (1997).

${ }^{9}$ E. Betzig, J. K. Trautman, T. D. Harris, J. S. Weiner, and R. L. Kostelak, Science 251, 1468 (1991).

${ }^{10}$ D. Gershoni, M. Katz, W. Wegscheider, L. N. Pfeiffer, R. A. Logan, and K. West, Phys. Rev. B 50, 8930 (1994).

${ }^{11}$ J. F. Ryan, A. C. Maciel, C. Kiener, L. Rota, K. Turner, J. M. Freyland, U. Marti, D. Martin, F. Morier-Gemoud, and F. K. Reinhart, Phys. Rev. B 53, R4 225 (1996).

${ }^{12}$ H. Hillmer, A. Forchel, and C. W. Tu, Phys. Rev. B 45, 1240 (1992), and references therein.

${ }^{13}$ M. A. Tamor and J. P. Wolfe, Phys. Rev. Lett. 44, 1703 (1980). 